\title{
Current and Innovative Pharmacological Options to Treat Typical and Atypical Trigeminal Neuralgia
}

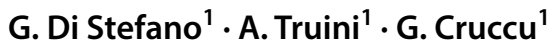

Published online: 3 September 2018

(c) The Author(s) 2018

\begin{abstract}
Trigeminal neuralgia is a representative neuropathic facial pain condition, characterised by unilateral paroxysmal pain in the distribution territory of one or more divisions of the trigeminal nerve, triggered by innocuous stimuli. A subgroup of patients with trigeminal neuralgia [TN (previously defined as atypical TN)] also suffer from concomitant continuous pain, i.e. a background pain between the paroxysmal attacks. The aim of this review is to provide current, evidence-based, knowledge about the pharmacological treatment of typical and atypical TN, with a specific focus on drugs in development. We searched for relevant papers within PubMed, EMBASE, the Cochrane Database of Systematic Reviews and the Clinical Trials database (ClinicalTrials.gov), taking into account publications up to February 2018. Two authors independently selected studies for inclusions, data extraction, and bias assessment. Carbamazepine and oxcarbazepine are the first-choice drugs for paroxysmal pain. When sodium channel blockers cannot reach full dosage because of side effects, an add-on treatment with lamotrigine or baclofen should be considered. In patients with atypical TN, both gabapentin and antidepressants are expected to be efficacious and should be tried as an add-on to oxcarbazepine or carbamazepine. Although carbamazepine and oxcarbazepine are effective in virtually the totality of patients, they are responsible for side effects causing withdrawal from treatment in an important percentage of cases. A new, better tolerated, Nav1.7 selective state-dependent, sodium channel blocker (vixotrigine) is under development. Future trials testing the effect of combination therapy in patients with TN are needed, especially in patients with concomitant continuous pain and in TN secondary to multiple sclerosis.
\end{abstract}

Electronic supplementary material The online version of this article (https://doi.org/10.1007/s40265-018-0964-9) contains supplementary material, which is available to authorized users.

G. Cruccu

giorgio.cruccu@uniroma1.it

1 Department of Human Neuroscience, Sapienza University, viale Università 30, 00185 Rome, Italy

\section{Key Points}

Carbamazepine and oxcarbazepine are the first-choice drugs for paroxysmal pain in patients with trigeminal neuralgia.

These drugs, effective in virtually all patients, produce side effects causing withdrawal from treatment in about $20 \%$ of cases.

A new, better tolerated, Nav1.7 selective state-dependent, sodium channel blocker is under development.

Currently there is a lack of evidence for most effective medications in patients affected by TN with concomitant continuous pain; these patients may benefit from medications used for neuropathic pain. 


\section{Introduction}

Trigeminal neuralgia (TN) is a representative neuropathic facial pain condition, characterised by unilateral paroxysmal pain described as stabbing or electric shocklike, in the distribution territory of one or more divisions of the trigeminal nerve and triggered by innocuous stimuli [1].

According to the new classification and diagnostic grading of TN issued by the International Association for the Study of Pain (IASP), TN is distinguished in three diagnostic categories-classical, caused by vascular compression producing anatomical changes in the trigeminal nerve root-secondary, due to an identifiable underlying neurologic disease,_-idiopathic, when even after MRI or other investigation, the aetiology of $\mathrm{TN}$ remains unclear (Table 1) [2].

Regardless of the aetiology, the primary mechanism of paroxysmal pain is the same, i.e. a focal demyelination of primary trigeminal afferents near the entry of the trigeminal root into the pons, making the axons hyper-excitable and increasing the susceptibility to ectopic excitation, ephaptic transmission, and high-frequency discharges [3-5].

$\mathrm{TN}$ has an annual incidence of three to five per 100,000. It is more common in women than men (age adjusted ratio: 1.74:1) and in people aged 50-69 years $[6,7]$.
In virtually the entire population of patients with $\mathrm{TN}$, at least one trigger capable of provoking a paroxysm can be identified. In a recent study, provocation of paroxysmal pain by various trigger manoeuvres was reported by 136 of the 140 patients. The most frequent manoeuvres were gentle touching of the face and talking. Trigger zones were predominantly reported in the perioral and nasal region and were variable in size [8]. These data are coherent with the use of trigger factor as an essential diagnostic feature for a clinically established diagnosis of TN [2]. Patients with TN may suffer from different types of pain, ranging from single attacks to a series of prolonged attacks, and it was suggested that these pain characteristics can vary over time [9].

Traditionally, autonomic symptoms such as tearing and rhinorrhoea have not been associated with TN. However, it is now known that a large proportion of TN patients have autonomic symptoms [10].

A subgroup of patients with TN also suffer from concomitant continuous pain (CCP), described as dull, burning or aching [11]. This condition has been described with several definitions, including atypical TN and TN type 2; the International Headache Society Classification (ICHD) [1] defined this relatively uncommon type of TN as TN with concomitant continuous facial pain. The presence of continuous pain is not related to aetiology and may occur in idiopathic, classic, or secondary TN. Background pain

Table 1 Trigeminal neuralgia diagnostic criteria

IASP criteria

Definition

Classification

ICHD criteria

Criteria

Classification
TN is orofacial pain restricted to one or more divisions of the trigeminal nerve. With the exception of TN caused by MS, the pain affects one side of the face. It is abrupt in onset and typically lasts only a few seconds ( 2 min at maximum). Patients may report their pain as arising spontaneously, but these pain paroxysms can always be triggered by innocuous mechanical stimuli or movements. Patients usually do not experience pain between paroxysms. If they do report additional continuous pain, in the same distribution and in the same periods as the paroxysmal pain, they are considered to have TN with continuous pain

Classical TN: caused by vascular compression of the trigeminal nerve root resulting in morphological changes of the root Secondary TN: caused by major neurological disease, e.g. a tumor of the cerebellopontine angle or MS Idiopathic TN: no apparent cause

A. At least three attacks of unilateral facial pain fulfilling criteria B and C

B. Occurring in one or more divisions of the trigeminal nerve, with no radiation beyond the trigeminal distribution

C. Pain has at least three of the following four characteristics:

1. recurring in paroxysmal attacks lasting from a fraction of a second to $2 \mathrm{~min}$

2. severe intensity

3. electric shock-like, shooting, stabbing or sharp in quality

4. precipitated by innocuous stimuli to the affected side of the face

D. No clinically evident neurological deficit

E. Not better accounted for by another ICHD-3 diagnosis

13.1.1.1 Classical TN (classical TN, purely paroxysmal; classical TN with concomitant continuous pain)

13.1.1.2 Secondary TN (TN attributed to MS; TN attributed to space-occupying lesion; TN attributed to other cause

13.1.1.3 Idiopathic TN (idiopathic TN, purely paroxysmal; idiopathic TN with concomitant continuous pain

IASP International Association for the Study of Pain, ICHD International Classification of Headache Disorders, $M S$ multiple sclerosis, $T N$ trigeminal neuralgia 
distribution coincides with that of the paroxysmal pain, and fluctuations of its intensity parallel in time those of the paroxysmal pain $[12,13]$. A prevalence three times higher in women than in men was reported [11]. In a cohort of 158 patients with $\mathrm{TN}$, continuous pain developed within a mean period of 1.5 years since the disease onset, thus suggesting that this kind of pain is not a consequence of a long duration of stabbing pain [11]. The mechanisms underlying continuous pain, as opposed to paroxysmal pain, are not fully understood, with implications for treatment. There is the evidence that continuous and paroxysmal pain may improve differently after microvascular decompression, thus supporting the hypothesis that the mechanisms responsible for the two pain components may be different [14-17]. Central mechanisms [18] and progressive root damage due to compression [19] have been proposed as possible factors.

Carbamazepine (CBZ) and oxcarbazepine (OXC) are the first-choice medical treatment in TN. They have the same mechanism of action, the blockade of voltage gated sodium channel in a frequency dependent manner, resulting in the stabilization of hyperexcited neural membranes and in the inhibition of repetitive firing. In patients with purely paroxysmal pain, CBZ and OXC are effective in virtually the entire patient population. However, they produce side effects to a level that cause interruption of treatment or a dosage reduction in $23 \%$ of patients [20], making necessary the development of new, more selective sodium channel blockers. Conversely in patients with $\mathrm{CCP}$, the efficacy of CBZ and $\mathrm{OXC}$ may drop, thus suggesting the opportunity to test the efficacy of different drug categories. A wide range of drugs has been investigated in TN, but the scientific literature highlighted the need of high quality clinical trials in TN [21].

The aim of this review, based on a systematic search of relevant literature, is to provide current, evidence-based, knowledge about the pharmacological treatment of typical and atypical TN, with a specific focus on drugs in development, such as botulinum toxin A and new, more selective sodium channel blockers.

\section{Search Process}

We searched for relevant papers within the PubMed, EMBASE and the Cochrane Database of Systematic Reviews, taking into account publications up to February 2018. All searches used the following synonyms for $\mathrm{TN}$ : trigeminal neuralgia and tic douloureux. The primary search was supplemented by a secondary search using the bibliographies of the retrieved articles. Only full-length, original communications including open-label studies were considered, and the search was limited to English language publications. Clinical trials database (ClinicalTrial.gov) has been checked in order to include in the analysis studies currently in progress. The review process was carried out independently by two reviewers and only publications independently approved by the two authors were taken into account (Supplementary Material 1). The authors independently assessed the quality of the individual trials during data extraction. Inclusion criteria were the following: trials including patients with a diagnosis of typical or atypical $\mathrm{TN}$, including classical, idiopathic and secondary $\mathrm{TN}$, and a minimum sample of 10 patients.

\section{Drugs in Classical or Idiopathic Trigeminal Neuralgia}

\subsection{First-line Treatment}

CBZ and OXC are the first-line treatment in TN. Their effect is related to the blockade of voltage-sensitive sodium channels in a frequency dependent manner, resulting in the stabilization of hyperexcited neural membranes and inhibition of repetitive firing.

Systematic reviews and randomised controlled trials, including 147 patients [22-29], demonstrated the efficacy of CBZ compared to placebo, with a number-needed-totreat (NNT) to obtain pain relief of 1.7-1.8. However, CBZ showed a number-needed-to-harm (NNH) of 3.4 for minor and 24 for severe adverse events [24]. In the study of Killian and Fromm [26], with a maximum daily dose of $1000 \mathrm{mg}, 19$ of 27 participants had a complete or very good response with CBZ compared with minimal or no response with placebo on a 5-day treatment. Nicol [27], using a cross-over design and a maximum daily dose of $2400 \mathrm{mg}$, reported that 15 of 20 participants randomised to initial CBZ had a good or excellent response after 14 days' treatment, compared with 6 of 24 reporting good or excellent response who started on placebo. Superiority of CBZ was also reported by Rockliff and Davis [28] in a small sample of patients with three days' treatment with CBZ. In the study from Rasmussen and Riishede [29], after 5 days' treatment, 46 of 55 patients with TN had good effect on CBZ, compared with 8 of 55 on placebo. Campbell and colleagues [25] reported a mean fall in maximum pain intensity of 58\% after 2 weeks treatment with CBZ 400-800 mg daily compared to $26 \%$ with placebo (Supplementary Material 1).

Compared to CBZ, OXC showed a similar efficacy in reducing pain attacks but a greater tolerability and a lower potential for drug interaction [30]. However, data from full randomised controlled trials are not available, thus precluding the NNT and NNH calculation.

A pilot study on OXC with extended-release formulation is currently in progress (ClinicalTrials.gov Identifier: NCT03374709). 


\subsection{Alternative Treatments}

\subsubsection{Lamotrigine}

Lamotrigine acts at level of the voltage-sensitive sodium channels, stabilises neural membranes and inhibits the release of excitatory neurotransmitters. Two systematic reviews [23, 31] identified a small double-blind crossover randomised controlled trial comparing lamotrigine versus placebo in 14 patients receiving $\mathrm{CBZ}$ or phenytoin. Patients continued to take a steady dose of CBZ or phenytoin throughout the trial over a 31-day period. Each arm of the trial lasted 2 weeks with an intervening 3-day washout period. The maintenance dose of lamotrigine was $400 \mathrm{mg}$. This study showed that lamotrigine in combination with CBZ or phenytoin was slightly more effective than placebo. The adverse reactions with both lamotrigine and placebo were predominantly dose-dependent effects on the central nervous system (CNS). One patient withdrew from the study due to severe pain during the placebo arm of the trial [32].

A crossover study involving 21 patients with $\mathrm{TN}$ compared lamotrigine $(400 \mathrm{mg})$ with CBZ (1200 mg) [33]. CBZ reduced pain in $90.5 \%$ (19/21) and lamotrigine in $62 \%$ $(13 / 21)$ of the patients using both visual analogue scale (VAS) and verbal rating scale. The reported side effects were headache, dizziness and skin rash.

\subsubsection{Baclofen}

Two studies tested the efficacy of baclofen in TN [34, 35]. This drug is a $\mathrm{GABA}_{\mathrm{B}}$ receptor agonist and depresses excitatory neurotransmission. Baclofen was superior to placebo in reducing the number of painful paroxysms in a randomised controlled trial including ten participants; baclofen significantly decreased the number of painful paroxysms in seven patients [34]. A double-blind crossover trial in 15 patients showed that L-baclofen was more effective than five times as much racemic baclofen in nine patients. Six of these nine patients have continued pain-free on L-baclofen for 4-17 months (mean, 10 months). L-baclofen was much better tolerated than racemic baclofen [35]. However, these studies showed several limitations, such as the small sample of patients and the short duration of treatment, so the results must be interpreted with caution.

\subsubsection{Pimozide, Tizanidine and Tocainide}

One systematic review [36] identified three randomised controlled trials comparing pimozide [37], tizanidine [38] and tocainide [39] with CBZ. Pimozide was more effective than CBZ in a double-blind crossover 24-week trial including 48 patients suffering from refractory $\mathrm{TN}$ but significant side effects of this neuroleptic drug, including CNS disturbances, hand tremors and memory impairment were reported [37]. The effect of tizanidine, a centrally acting alpha-adrenergic agonist, in comparison with $\mathrm{CBZ}$ was tested in a very small sample of patients. After individual titration of tizanidine and CBZ, the maximum daily doses were $18 \mathrm{mg}$ and $900 \mathrm{mg}$, respectively, and the difference was not statistically significant [38]. Tocainide, a sodium channel blocker with anti-arrhythmic action, was tested in 12 participants in a double-blind cross-over study for 2 weeks, but significant side effects limited its use [39].

\subsubsection{Calcium Channel Blockers}

The efficacy of calcium channel blockers, gabapentin and pregabalin, has been assessed in small controlled or openlabel studies. These drugs exert their pharmacodynamic effect by modulating voltage-gated calcium channels and thus reducing release of excitatory neurotransmitters.

One systematic review and meta-analysis [40] identified 16 randomised controlled trials, all published in Chinese, on the efficacy of gabapentin in comparison with CBZ in the treatment of TN. 1331 patients were included and VAS score was used to assess the effect of treatment. The total effective rate of gabapentin therapy group was similar with CBZ therapy group and the adverse reaction rate of gabapentin therapy group was significantly lower than that of CBZ. However, the authors concluded that the trials are all poor in terms of methodological quality [40].

Pregabalin was not tested in randomised controlled trials. An open-label study including 53 patients showed the efficacy of pregabalin in reducing TN by over $50 \%$ in $74 \%$ of patients [41]. Two observational studies, including 65 patients, proved the efficacy of pregabalin in monotherapy $(n=36)$ or add-on $(n=29)$ for 12 weeks. However, according to the inclusion criteria, patients with an atypical facial pain might also have been included [42, 43].

In an open-label crossover trial involving 22 patients with refractory $\mathrm{TN}$ using lamotrigine and pregabalin together with CBZ, pregabalin showed a comparable efficacy and a better patient tolerance than lamotrigine [44].

\subsubsection{Levetiracetam}

Among anticonvulsants, open label studies investigated the efficacy of levetiracetam. A pilot study investigated the efficacy and tolerability of this drug in 10 patients with TN over a period of 10 weeks. Patients were treated with up to $4000 \mathrm{mg}$ daily and $40 \%$ reported an improvement of 50\%-90\% [45]. In an observational trial, including 23 patients with refractory $\mathrm{TN}$, levetiracetam ( $3-4 \mathrm{~g} /$ day) for 16 weeks decreased the number of daily attacks by $62.4 \%$ [46]. 


\subsubsection{Eslicarbazepine}

Eslicarbazepine, a third-generation antiepileptic drug belonging to the dibenzazepine group, targets the voltagegated sodium channels and is currently approved as adjunct therapy for focal seizures. A recent retrospective, open-label, multicentric, intention-to-treat study assessed the efficacy and safety of this drug in patients with TN. Eighteen participants were included; the dose of eslicarbazepine ranged between 200 and $1200 \mathrm{mg} /$ day. Responder rate was $88.9 \%$; $71 \%$ of patients presented some adverse events and four patients discontinued the treatment [47].

\subsubsection{Local Anaesthetics}

Two randomised controlled trials investigated the effect of local anaesthesia injected into trigger area [48, 49]. These studies, combining the peripheral analgesic block with ropivacaine and CBZ or gabapentin, showed improved outcome. In the randomised controlled trial combining the peripheral analgesic block with ropivacaine and CBZ in 45 patients, the association protocol resulted in a significant reduction in pain intensity, number of daily pain paroxysms and daily dose of CBZ intake, when compared with CBZ in monotherapy [48].

In the randomised controlled trial in 36 patients combining gabapentin with ropivacaine injection into trigger areas showed improved pain control and quality of life [49]. The association treatment was safe, without side effects and resulted in an important clinical benefit with an improvement of the functional health status of TN patients when compared with gabapentin alone. A reduction of VAS score after 7 and 28 days of treatment was reported, and this effect was still present 6 and 12 months later. The NNT (gabapentin + ropivacaine vs gabapentin protocols) to obtain 1 gabapentin + ropivacaine-treated patient with at least $50 \%$ pain relief was 1.71 (day 7) and 2.40 (day 28).

Two randomised controlled trials tested the short-term effect of topical $8 \%$ lidocaine versus placebo in TN [50, 51]. In the study of Kanai et al. [50], including 25 patients with TN involving the second division, those given an $8 \%$ spray of lidocaine as opposed to saline had a statistically significant decrease in pain. The effect of treatment persisted for a mean of $4 \mathrm{~h}$ without serious adverse reactions. In the study of Niki et al. [51], including 24 patients with TN and severe intraoral pain, the application on the painful areas of $8 \%$ lidocaine significantly reduced the NRS score of paroxysmal pain for a mean of $3 \mathrm{~h}$, without serious side effects. However, these studies showed several criticisms and the findings must be interpreted with care.

\subsubsection{Sumatriptan}

Recent studies tested the effect of 5-HT1A/1B/1D receptor agonist on pain relief in patients with $\mathrm{TN}$. These drugs may inhibit vasodilation and inflammation near the demyelinated trigeminal root. Two randomised controlled trials tested the effect of subcutaneous injection of sumatriptan $3 \mathrm{mg}$ and the oral administration of $50 \mathrm{mg}$ twice daily [52, 53]. Fifteen minutes after injection of sumatriptan, the baseline VAS decreased from 8.3 to $2.4 \mathrm{~cm}$. At the end of oral treatment, the VAS was significantly decreased, and this effect persisted after treatment discontinuation for a further week. However, the side effects related to a long-term use, including a triptan overuse headache, exclude the use of sumatriptan in the long-term treatment of TN.

\subsection{Intravenous Drugs for Acute Exacerbations}

In a randomised controlled trial including 20 patients with intractable $\mathrm{TN}$, a single dose of intravenous lidocaine $(5 \mathrm{mg} /$ $\mathrm{kg}$ over $60 \mathrm{~min}$ ) was superior in reducing pain intensity compared to placebo during the first $24 \mathrm{~h}$ after the infusion [54].

\subsection{Drugs Under Development}

\subsubsection{Vixotrigine}

A new Nav1.7 selective state-dependent, sodium channel blocker (vixotrigine) is under development. Nav1.7, a major sodium receptor in the nociceptive system, is not located in the brain, thus preventing any side effects associated with depression of CNS excitability [55]. A Phase 1 study demonstrated the good tolerability of vixotrigine, administered at therapeutic doses without lengthy titration. The new drug was tested in a double-blind, placebo-controlled, randomised withdrawal Phase 2a trial, including 67 patients with classical and idiopathic TN [21]. After a 7-day run-in phase, eligible patients received open-label, vixotrigine $150 \mathrm{mg} 3$ times per day, orally, for 21 days. Patients who met at least one response criteria were then randomly assigned (1:1) to vixotrigine or placebo for up to 28 days in a double-blind phase. Although the primary endpoint of treatment failure was not significantly lower in the vixotrigine group than in the placebo group, significant treatment differences versus placebo in secondary endpoints were found, including time to treatment failure, number of paroxysms and average daily pain score. The new drug was well tolerated, and no severe or serious adverse events were reported. A Phase 3 placebo-controlled, double-blind randomised withdrawal study is currently in progress (ClinicalTrials.gov Identifier: NCT03070132). 


\subsection{Presurgical Procedures}

\subsubsection{Botulinum Toxin A}

Botulinum toxin type A (BTX-A), an exotoxin released by the gram-positive bacterium Clostridium botulinum, is supposed to block the TRPV1 receptor of unmyelinated C fibre terminals and limit the release of substance $\mathrm{P}$, calcitonin gene-related peptide and glutamate from presynaptic terminals of the primary sensory neurons.

A systematic review [56] identified 4 randomised controlled trials, on 178 patients, testing the effect of BTX-A in patients with TN [57-60]. The total dosage administered varies from $25 \mathrm{U}$ to $75 \mathrm{U}$ and the number of injections from 8 to 20. The injections were administered intradermally and/or submucosally where pain was experienced according to the patient's description. The overall effect favoured BTX-A versus placebo in terms of proportion of responders; paroxysms frequency per day was significantly lower for BTX-A group. The duration of effect was relatively long (at least 3 months). Adverse events included transient facial weakness, oedema and haematoma at the site of injection. Despite these encouraging findings, future studies assessing the optimal dose, duration of the therapeutic efficacy, adverse events, the time and indications for repeat injection are required. To our knowledge, no data are available about the effect of repeated BTX-A injections in $\mathrm{TN}$.

A clinical trial comparing intradermal/submucosal injection and intra-masseter injection of BTX-A is currently in progress (ClinicalTrials.gov Identifier: NCT03331913).

\subsubsection{Intranasal Non-inhaled Carbon Dioxide}

Recently, $\mathrm{CO}_{2}$ has been shown to be a modulator of activated nociceptive trigeminal neurons [61]. Based on animal model, an antinociceptive effect of intranasal $\mathrm{CO}_{2}$ by activation of mucosal primary trigeminal afferents through a decreased mucosal $\mathrm{pH}$ was postulated [62]. A controlled, randomised, parallel-group study investigated the effects of intranasal $\mathrm{CO}_{2}$ on TRPV1-mediated trigeminal pain in healthy volunteers. Only mild modulatory effect of intranasal insufflation of $\mathrm{CO}_{2}$ at flow rates of $1 \mathrm{~L} / \mathrm{min}$ was found, and the clinical utility seemed limited since changes in pain ratings were therapeutically irrelevant [63]. A Phase 2 placebo-controlled, single-blind study to evaluate the safety and efficacy of nasal $\mathrm{CO}_{2}$ has been conducted in patients with classical TN. All subjects received 3 doses of active and 3 doses of placebo. A single dose consisted of a 60 -second delivery of $\mathrm{CO}_{2}$ or placebo. The primary outcome was pain relief assessed on VAS. Recruitment phase is completed; however, the study findings have not yet been published (ClinicalTrials.gov Identifier: NCT02473016).

\section{Drugs in Secondary Trigeminal Neuralgia}

No randomised controlled trials were found in patients with secondary TN. The existing studies based on CBZ, OXC, eslicarbazepine, lamotrigine, gabapentin, pregabalin, topiramate and misoprostol have an open label design and include small sample of patients with multiple sclerosis (MS) [47, 64-78]. Only few studies provided details about the type of MS and the TN diagnostic criteria [78]. One study used a VAS and recorded both intensity and number of attacks [75, 78]. These case series reported the potential efficacy of lamotrigine as monotherapy or in association with gabapentin or CBZ, topiramate and gabapentin. Three studies reported the efficacy of misoprostol (a prostaglandin-E1-analogue) in a total of 27 patients with TN secondary to MS [74-76]. According to the international guidelines [22], there is insufficient evidence to support or refute the effectiveness of any medication in treating pain in TN secondary to MS.

\section{Drugs in Trigeminal Neuralgia with Concomitant Continuous Pain}

No clinical trials assessing pharmacological treatment of $\mathrm{CCP}$ in TN have been conducted. Different studies clearly demonstrated that continuous pain is associated with poor medical and surgical outcome [11, 14, 43]. Recently, in a prospective study including 158 patients with TN (also including patients with $\mathrm{CCP}$ ), the prevalence of responders to sodium channel blockers was lower in the group with also CCP [10]. On the basis of these data, constant pain is considered a predictor of poor treatment response. No study has assessed the drug effect in reducing constant and paroxysmal pain intensity separately. Because CBZ and OXC are extremely efficacious in increasing the refractory period of action potentials, they act on the high frequency discharges that characterise the paroxysms of TN. Usually, in patients with also continuous pain mediated by other pathophysiological mechanisms, a monotherapy with sodium channel blocker is not sufficient to control pain and other drugs are usually needed. As far as we know, calcium channel blockers and antidepressants, whose efficacy has already been proven in the treatment of neuropathic continuous pain due to several aetiologies, have never been systematically tested in TN patients with CCP. Future randomised controlled trials assessing these drugs as add-on treatment in TN with CCP are required.

\section{Expert Opinion}

Based on evidence [22], CBZ (400-1200 mg/day) and OXC (900-1800 mg/day) are the first-choice medical treatment in TN. OXC should be preferred for better 
tolerability and the decreased potential for drugs interactions. These drugs are effective in most patients and the development of late resistance only occurred in a very small minority of patients. However, the side effects cause withdrawal from treatment in an important percentage of patients [20]. Common initial side effects include drowsiness, nausea, dizziness, diplopia, ataxia and elevation of transaminases. Hyponatraemia occurs in $6-8 \%$ of patients; sodium levels are dose related and should be monitored during the treatment, especially when high dosage is used. Patients treated with diuretics may be more susceptible to developing sodium depletion [14]. The addition of sodium chloride capsules can be helpful in patients with persistent hyponatremia. Serious but uncommon side effects include allergic rash, hepatotoxicity, lymphadenopathy, systemic lupus erythematosus, Stevens-Johnson syndrome and aplastic anaemia. Specific contraindications are cardiac conduction problems or severe arrhythmias.

In a prospective, observational, exploratory survey of 161 patients with idiopathic TN, females treated with CBZ or OXC reported significantly more side effects than males [79]. Pharmacokinetic and pharmacodynamic differences are likely to be the reason for gender differences in reporting side effects.

The consumers' views on treatments used for TN was investigated by using a self-administered questionnaire distributed to 133 patients and 21 clinicians attending national support group meetings in the USA and UK [80]. All patients reported at least one side effect. The clinicians underestimated the number of side effects, but both groups agreed that drowsiness and cognitive impairment were the most disliked side effects.

A prospective study investigated the risk and genetic association of OXC-induced cutaneous adverse reactions, including Stevens-Johnson syndrome/toxic epidermal necrolysis, in Asian populations. The authors found that HLA-B*15:02 was significantly associated with OXC-Stevens-Johnson syndrome in Chinese and Thai populations [81].

CBZ is a potent inducer of CYP3A4 and other oxidative enzyme systems in the liver, and it may also increase glucuronyl transferase activity, leading to a number of clinically relevant drug interactions. OXC, a keto-analogue of CBZ, rapidly converted into its pharmacologically active metabolite, should be preferred for the better tolerability and the decreased potential for drugs interactions.

A recent meta-analysis investigating the teratogenic effects of different antiepileptic drugs showed that children exposed to CBZ were at a higher risk of malformation than children born to women without, and women with untreated epilepsy [82].

Vixotrigine, a new sodium channel blocker that is selective for the Nav1.7 receptor is under development and has promise of efficacy without inducing side effects related to CNS depression [21].

Lamotrigine is considered a second-line treatment in patients with TN. Potential side effects of lamotrigine include dizziness, nausea, blurred vision and ataxia. Approximately $7-10 \%$ of patients will report a skin rash during the first 48 weeks of therapy [32]. The dose of lamotrigine must be increased slowly in order to avoid skin rash.

In patients with refractory $\mathrm{TN}$, or in the event of withdrawal due to side effects, surgery should at least be proposed and discussed with the patient.

In refractory TN, BTX-A is a promising alternative treatment option that might spare the need for surgical interventions [83]. Although it is reasonable that BTX-A primarily acts on constant pain, to our knowledge, no study has reported the effects of injections in a subgroup of patients with CCP until now.

During acute exacerbation, in-hospital treatment may be necessary for rehydration, management of hyponatraemia, titration of drugs, and, in selected case, lidocaine or fosphenytoin intravenous infusion, under specialist supervision and cardiac monitoring. Intravenous loading of fosphenytoin was reported in case series but no randomised controlled trials have been conducted until now [7, 84-86].

The first-line therapy in secondary TN is based on sodium channel blockers. In patients with MS-related TN, gabapentin, lamotrigine and topiramate represent other therapeutic options, but the quality of evidence is poor. In this patient category pharmacological treatment may potentiate some of the MS symptoms with a high dropout rate. Expert consensus suggests that baclofen may be useful in patients with MS who develop TN. Such patients are often taking baclofen already to reduce spasticity and may achieve control of symptoms without having to add CBZ. The main side effects of baclofen are transient sedation and loss of muscle tone. Abrupt discontinuation may cause seizures and hallucinations [87].

In patients with $\mathrm{TN}, \mathrm{CCP}$ is associated with poor medical and surgical outcome. In this condition, both calcium channel blockers (gabapentin and pregabalin) and antidepressants may be efficacious and should be tried as an add-on to OXC or CBZ. However, randomised, controlled, double-blinded trials are still lacking.

\section{Compliance with Ethical Standards}

Conflict of interest Giorgio Cruccu received a research grant, consulting fees and payments for lectures from Alfasigma, and consulting fees from Angelini and Biogen. Andrea Truini received consulting fees or payment for lectures from Alfasigma, Angelini, Grünenthal and Pfizer. Giulia Di Stefano has no conflicts to declare.

Funding None (GDS, AT \& GC). 
Open Access This article is distributed under the terms of the Creative Commons Attribution-NonCommercial 4.0 International License (http://creativecommons.org/licenses/by-nc/4.0/), which permits any noncommercial use, distribution, and reproduction in any medium, provided you give appropriate credit to the original author(s) and the source, provide a link to the Creative Commons license, and indicate if changes were made.

\section{References}

1. Headache Classification Committee of the International Headache Society (IHS). The International Classification of Headache Disorders, 3rd edition. Cephalalgia. 2018;38:1-211.

2. Cruccu G, Finnerup NB, Jensen TS, Scholz J, Sindou M, Svensson $\mathrm{P}$, et al. Trigeminal neuralgia: new classification and diagnostic grading for practice and research. Neurology. 2016;12(87):220-8.

3. Burchiel KJ. Abnormal impulse generation in focally demyelinated trigeminal roots. J Neurosurg. 1980;53:674-83.

4. Calvin WH, Devor M, Howe JF. Can neuralgias arise from minor demyelination? Spontaneous firing, mechanosensitivity, and afterdischarge from conducting axons. Exp Neurol. 1982;75:755-63.

5. Devor M, Amir R, Rappaport ZH. Pathophysiology of trigeminal neuralgia: the ignition hypothesis. Clin J Pain. 2002;18:4-13.

6. Katusic S, Beard CM, Bergstralh E, Kurland LT. Incidence and clinical features of trigeminal neuralgia, Rochester, Minnesota, 1945-1984. Ann Neurol. 1990;27:89-95.

7. Maarbjerg S, Di Stefano G, Bendtsen L, Cruccu G. Trigeminal neuralgia-diagnosis and treatment. Cephalalgia. 2017;37:648-57.

8. Di Stefano G, Maarbjerg S, Nurmikko T, Truini A, Cruccu G. Triggering trigeminal neuralgia. Cephalalgia. 2017;1:333102417721677.

9. Zakrzewska JM, Jensen TS. History of facial pain diagnosis. Cephalalgia. 2017;37:604-8.

10. Maarbjerg S, Gozalov A, Olesen J, Bendtsen L. Trigeminal neuralgia - a prospective systematic study of clinical characteristics in 158 patients. Headache. 2014;54(10):1574-82.

11. Maarbjerg S, Gozalov A, Olesen J, Bendtsen L. Concomitant persistent pain in classical trigeminal neuralgia — evidence for different subtypes. Headache. 2014;54:1173-83.

12. Sandell T, Eide PK. Effect of microvascular decompression in trigeminal neuralgia patients with or without constant pain. Neurosurgery. 2008;63:93-9.

13. Brisman R. Typical versus atypical trigeminal neuralgia and other factors that may affect results of neurosurgical treatment. World Neurosurg. 2013;79:649-50.

14. Cruccu G. Trigeminal Neuralgia. Continuum (Minneap Minn). 2017;23:396-420.

15. Zhang H, Lei D, You C, Mao BY, Wu B, Fang Y. The long-term outcome predictors of pure microvascular decompression for primary trigeminal neuralgia. World Neurosurg. 2013;79:756-62.

16. Sindou M, Leston J, Howeidy T, Decullier E, Chapuis F. Microvascular decompression for primary trigeminal neuralgia (typical or atypical). Long-term effectiveness on pain; prospective study with survival analysis in a consecutive series of 362 patients. Acta Neurochir (Wien). 2006;148:1235-45 (discussion 1245).

17. Maarbjerg S, Wolfram E, Gozalov A, Olesen J, Bendtsen L. Association between neurovascular contact and clinical characteristics in classical trigeminal neuralgia: a prospective clinical study using 3.0 Tesla MRI. Cephalalgia. 2015;35:1077-84.

18. Obermann M, Yoon MS, Ese D, Maschke M, Kaube H, Diener $\mathrm{HC}$, et al. Impaired trigeminal nociceptive processing in patients with trigeminal neuralgia. Neurology. 2007;69:835-41.
19. Burchiel KJ. A new classification for facial pain. Neurosurgery. 2003;53:1164-6.

20. Di Stefano G, La Cesa S, Truini A, Cruccu G. Natural history and outcome of 200 outpatients with classical trigeminal neuralgia treated with carbamazepine or oxcarbazepine in a tertiary centre for neuropathic pain. J Headache Pain. 2014;15:34.

21. Zakrzewska JM, Palmer J, Morisset V, Giblin GM, Obermann M, Ettlin DA, et al. Safety and efficacy of a Nav1.7 selective sodium channel blocker in patients with trigeminal neuralgia: a doubleblind, placebo-controlled, randomised withdrawal phase 2a trial. Lancet Neurol. 2017;16:291-300.

22. Cruccu G, Gronseth G, Alksne J, Argoff C, Brainin M, Burchiel K, American Academy of Neurology Society, European Federation of Neurological Society, et al. AAN-EFNS guidelines on trigeminal neuralgia management. Eur J Neurol. 2008;15:1013-28.

23. Zakrzewska JM, Linskey ME. Trigeminal neuralgia. BMJ Clin Evid. 2014;6:2014.

24. Wiffen PJ, Derry S, Moore RA, McQuay HJ. Carbamazepine for acute and chronic pain in adults. Cochrane Database Syst Rev. 2011;1:CD005451.

25. Campbell FG, Graham JG, Zilkha KJ. Clinical trial of carbamazepine (Tegretol) in trigeminal neuralgia. J Neurol Neurosurg Psychiatry. 1966;29:265-7.

26. Killian JM, Fromm GH. Carbamazepine in the treatment of neuralgia. Arch Neurol. 1968;19:129-36.

27. Nicol CF. A four year double-blind study of tegretol in facial pain. Headache. 1969;9:54-7.

28. Rockliff BW, Davis EH. Controlled sequential trials of carbamazepine in trigeminal neuralgia. Arch Neurol. 1966;15:129-36.

29. Rasmussen P, Riishede J. Facial pain treated with carbamazepin (Tegretol). Acta Neurol Scand. 1970;46:385-408.

30. Beydoun A. Safety and efficacy of oxcarbazepine: results of randomised, double-blind trials. Pharmacotherapy. 2000;20:152S-8S

31. Wiffen PJ, Derry S, Moore RA. Lamotrigine for acute and chronic pain. Cochrane Database Syst Rev. 2011;16:CD006044.

32. Zakrzewska JM, Chaudhry Z, Nurmikko TJ, Patton DW, Mullens EL. Lamotrigine (lamictal) in refractory trigeminal neuralgia: results from a double-blind placebo-controlled crossover trial. Pain. 1997;73:223-30.

33. Shaikh S, Yaacob HB, Abd Rahman RB. Lamotrigine for trigeminal neuralgia: efficacy and safety in comparison with carbamazepine. J Chin Med Assoc. 2011;74:243-9.

34. Fromm GH, Terrence CF, Chattha AS. Baclofen in the treatment of trigeminal neuralgia: double-blind study and long-term followup. Ann Neurol. 1984;15:240-4.

35. Fromm GH, Terrence CF. Comparison of $\mathrm{L}$-baclofen and racemic baclofen in trigeminal neuralgia. Neurology. 1987;37:1725-8.

36. Yang M, Zhou M, He L, Chen N, Zakrzewska JM. Non-antiepileptic drugs for trigeminal neuralgia. Cochrane Database Syst Rev. 2011;19:CD004029.

37. Lechin F, van der Dijs B, Lechin ME, Amat J, Lechin AE, Cabrera A, et al. Pimozide therapy for trigeminal neuralgia. Arch Neurol. 1989;46:960-3.

38. Vilming ST, Lyberg T, Lataste X. Tizanidine in the management of trigeminal neuralgia. Cephalalgia. 1986;6:181-2.

39. Lindström P, Lindblom U. The analgesic effect of tocainide in trigeminal neuralgia. Pain. 1987;28:45-50.

40. Yuan M, Zhou HY, Xiao ZL, Wang W, Li XL, Chen SJ, et al. Efficacy and safety of gabapentin vs. carbamazepine in the treatment of trigeminal neuralgia: a meta-analysis. Pain Pract. 2016. https://doi.org/10.1111/papr.12406.

41. Obermann M, Yoon MS, Sensen K, Maschke M, Diener HC, Katsarava Z. Efficacy of pregabalin in the treatment of trigeminal neuralgia. Cephalalgia. 2008;28:174-81.

42. Perez C, Navarro A, Saldaña MT, Martínez S, Rejas J. Patientreported outcomes in subjects with painful trigeminal neuralgia 
receiving pregabalin: evidence from medical practice in primary care settings. Cephalalgia. 2009;29:781-90.

43. Perez C, Saldaña MT, Navarro A, Martínez S, Rejas J. Trigeminal neuralgia treated with pregabalin in family medicine settings: its effect on pain alleviation and cost reduction. J Clin Pharmacol. 2009;49:582-90.

44. Rustagi A, Roychoudhury A, Bhutia O, Trikha A, Srivastava MV. Lamotrigine versus pregabalin in the management of refractory trigeminal neuralgia: a randomised open label crossover trial. J Maxillofac Oral Surg. 2014;13:409-18.

45. Jorns TP, Johnston A, Zakrzewska JM. Pilot study to evaluate the efficacy and tolerability of levetiracetam (Keppra) in treatment of patients with trigeminal neuralgia. Eur J Neurol. 2009;16:740-4.

46. Mitsikostas DD, Pantes GV, Avramidis TG, et al. An observational trial to investigate the efficacy and tolerability of levetiracetam in trigeminal neuralgia. Headache. 2010;50:1371-7.

47. Sanchez-Larsen A, Sopelana D, Diaz-Maroto I, Perona-Moratalla AB, Gracia-Gil J, García-Muñozguren S, et al. Assessment of efficacy and safety of eslicarbazepine acetate for the treatment of trigeminal neuralgia. Eur J Pain. 2018. https://doi.org/10.1002/ ejp.1192.

48. Lemos L, Fontes R, Flores S, Oliveira P, Almeida A. Effectiveness of the association between carbamazepine and peripheral analgesic block with ropivacaine for the treatment of trigeminal neuralgia. J Pain Res. 2010;25(3):201-12.

49. Lemos L, Flores S, Oliveira P, Almeida A. Gabapentin supplemented with ropivacain block of trigger points improves pain control and quality of life in trigeminal neuralgia patients when compared with gabapentin alone. Clin J Pain. 2008;24:64-75.

50. Kanai A, Suzuki A, Kobayashi M, Hoka S. Intranasal lidocaine $8 \%$ spray for second-division trigeminal neuralgia. Br J Anaesth. 2006;97:559-63

51. Niki Y, Kanai A, Hoshi K, Okamoto H. Immediate analgesic effect of $8 \%$ lidocaine applied to the oral mucosa in patients with trigeminal neuralgia. Pain Med. 2014;15:826-31.

52. Kanai A, Saito M, Hoka S. Subcutaneous sumatriptan for refractory trigeminal neuralgia. Headache. 2006;46:577-82.

53. Kanai A, Suzuki A, Osawa S, Hoka S. Sumatriptan alleviates pain in patients with trigeminal neuralgia. Clin J Pain. 2006;22:677-80.

54. Stavropoulou E, Argyra E, Zis P, Vadalouca A, Siafaka I. The effect of intravenous lidocaine on trigeminal neuralgia: a randomised double blind placebo controlled trial. ISRN Pain. 2014:853826. https://doi.org/10.1155/2014/853826.

55. Zakrzewska JM, Palmer J, Ettlin DA, Obermann M, Giblin GM, Morisset V, Tate S, Gunn K. Novel design for a phase IIa placebocontrolled, double-blind randomised withdrawal study to evaluate the safety and efficacy of CNV1014802 in patients with trigeminal neuralgia. Trials. 2013;23(14):402.

56. Morra ME, Elgebaly A, Elmaraezy A, Khalil AM, Altibi AM, $\mathrm{Vu} \mathrm{TL}$, et al. Therapeutic efficacy and safety of botulinum toxin a therapy in trigeminal neuralgia: a systematic review and meta-analysis of randomised controlled trials. J Headache Pain. 2016;17:63.

57. Wu CJ, Lian YJ, Zheng YK, Zhang HF, Chen Y, Xie NC, et al. Botulinum toxin type A for the treatment of trigeminal neuralgia: results from a randomised, double-blind, placebo-controlled trial. Cephalalgia. 2012;32:443-50.

58. Zhang H, Lian Y, Ma Y, Chen Y, He C, Xie N, et al. Two doses of botulinum toxin type $\mathrm{A}$ for the treatment of trigeminal neuralgia: observation of therapeutic effect from a randomised, double-blind, placebo-controlled trial. J Headache Pain. 2014;15:65.

59. Zúñiga C, Piedimonte F, Díaz S, Micheli F. Acute treatment of trigeminal neuralgia with onabotulinum toxin A. Clin Neuropharmacol. 2013;36:146-50.
60. Shehata HS, El-Tamawy MS, Shalaby NM, Ramzy G. Botulinum toxin type A: could it be an effective treatment option in intractable trigeminal neuralgia? J Headache Pain. 2013;14:92.

61. Vause C, Bowen E, Spierings E, Durham P. Effect of carbon dioxide on calcitonin gene-related peptide secretion from trigeminal neurons. Headache. 2007;47:1385-97.

62. Tzabazis AZ, Niv SH, Manering NA, Klyukinov M, Cuellar JM, Bhatnagar A, et al. Trigeminal antihyperalgesic effect of intranasal carbon dioxide. Life Sci. 2010;87:36-41.

63. Jürgens TP, Reetz R, May A. No relevant modulation of TRPV1mediated trigeminal pain by intranasal carbon dioxide in healthy humans. J Headache Pain. 2013;14:33.

64. Espir ML, Millac P. Treatment of paroxysmal disorders in multiple sclerosis with carbamazepine (Tegretol). J Neurol Neurosurg Psychiatry. 1970;33:528-31.

65. Ramsaransing G, Zwanikken C, De Keyser J. Worsening of symptoms of multiple sclerosis associated with carbamazepine. BMJ. 2000;320:1113.

66. Lunardi G, Leandri M, Albano C, Cultrera S, Fracassi M, Rubino $\mathrm{V}$, et al. Clinical effectiveness of lamotrigine and plasma levels in essential and symptomatic trigeminal neuralgia. Neurology. 1997;48:1714-7.

67. Leandri M, Lundardi G, Inglese M, Messmer-Uccelli M, Mancardi GL, Gottlieb A, et al. Lamotrigine in trigeminal neuralgia secondary to multiple sclerosis. J Neurol. 2000;247:556-8.

68. Khan OA. Gabapentin relieves trigeminal neuralgia in multiple sclerosis patients. Neurology. 1998;51:611-4.

69. Solaro C, Lunardi GL, Capello E, Inglese M, Messmer Uccelli M, Uccelli A, et al. An open-label trial of gabapentin treatment of paroxysmal symptoms in multiple sclerosis patients. Neurology. 1998;51:609-11.

70. Solaro C, Boehmker M, Tanganelli P. Pregabalin for treating paroxysmal painful symptoms in multiple sclerosis: a pilot study. J Neurol. 2009;256:1773-4.

71. Zvartau-Hind M, Din MU, Gilani A, Lisak RP, Khan OA. Topiramate relieves refractory trigeminal neuralgia in MS patients. Neurology. 2000;55:1587-8.

72. Solaro C, Uccelli MM, Brichetto G, Gaspperini C, Mancardi G. Topiramate relieves idiopathic and symptomatic trigeminal neuralgia. J Pain Symptom Manag. 2001;21:367-8.

73. D’Aleo G, Sessa E, Di Bella P, Rifici C, Restivo DA, Bramanti P. Topiramate modulation of R3 nociceptive reflex in multiple sclerosis patients suffering paroxysmal symptoms. J Neurol. 2001;248:996-9.

74. Reder AT, Arnason BG. Trigeminal neuralgia in multiple sclerosis relieved by a prostaglandin E analogue. Neurology. 1995;45:1097-100.

75. DMKG study group. Misoprostol in the treatment of trigeminal neuralgia associated with multiple sclerosis. J. Neurol. 2003;250:542-5.

76. Pfau G, Brinkers M, Treuheit T, Kretzschmar M, Sentürk M, Hachenberg T. Misoprostol as a therapeutic option for trigeminal neuralgia in patients with multiple sclerosis. Pain Med. 2012:13:1377-8.

77. Solaro C, Messmer Uccelli M, Uccelli A, Uccelli A, Leandri M, Mancardi GL. Low-dose gabapentin combined with either lamotrigine or carbamazepine can be useful therapies for trigeminal neuralgia in multiple sclerosis. Eur Neurol. 2000;44:45-8.

78. Zakrzewska JM, Wu J, Brathwaite TS. A systematic review of the management of trigeminal neuralgia in patients with multiple sclerosis. World Neurosurg. 2018;111:291-306.

79. Besi E, Boniface DR, Cregg R, Zakrzewska JM. Comparison of tolerability and adverse symptoms in oxcarbazepine and carbamazepine in the treatment of trigeminal neuralgia and 
neuralgiform headaches using the Liverpool Adverse Events Profile (AEP). J Headache Pain. 2015;16:563.

80. Zakrzewska JM. Consumer views on management of trigeminal neuralgia. Headache. 2001;41:369-76.

81. Chen CB, Hsiao YH, Wu T, Hsih MS, Tassaneeyakul W, Jorns TP. Risk and association of HLA with oxcarbazepine-induced cutaneous adverse reactions in Asians. Neurology. 2017;88:78-86.

82. Weston J, Bromley R, Jackson CF, Adab N, Clayton-Smith J, Greenhalgh J, et al. Monotherapy treatment of epilepsy in pregnancy: congenital malformation outcomes in the child. Cochrane Database Syst Rev. 2016;11:CD010224.

83. Cruccu G, Truini A. Refractory trigeminal neuralgia. Non-surgical treatment options. CNS Drugs. 2013;27:91-6.
84. Cheshire WP. Fosphenytoin: an intravenous option for the management of acute trigeminal neuralgia crisis. J Pain Symptom Manag. 2001;21:506-10.

85. Tate R, Rubin LM, Krajewski KC. Treatment of refractory trigeminal neuralgia with intravenous phenytoin. Am J Health Syst Pharm. 2011;68:2059-61.

86. Vargas A, Thomas K. Intravenous fosphenytoin for acute exacerbation of trigeminal neuralgia: case report and literature review. Ther Adv Neurol Disord. 2015;8:187-8.

87. Zakrzewska JM, Linskey ME. Trigeminal neuralgia. BMJ. 2015;350:h1238. 